ESAIM: COCV 18 (2012) 693-711

DOI: $10.1051 / \mathrm{cocv} / 2011167$
ESAIM: Control, Optimisation and Calculus of Variations

www.esaim-cocv.org

\title{
OPTIMAL CONVEX SHAPES FOR CONCAVE FUNCTIONALS
}

\author{
Dorin BuCur ${ }^{1}$, Ilaria Fragalì ${ }^{2}$ AND Jimmy LAmbOLEY ${ }^{3}$
}

\begin{abstract}
Motivated by a long-standing conjecture of Pólya and Szegö about the Newtonian capacity of convex bodies, we discuss the role of concavity inequalities in shape optimization, and we provide several counterexamples to the Blaschke-concavity of variational functionals, including capacity. We then introduce a new algebraic structure on convex bodies, which allows to obtain global concavity and indecomposability results, and we discuss their application to isoperimetric-like inequalities. As a byproduct of this approach we also obtain a quantitative version of the Kneser-Süss inequality. Finally, for a large class of functionals involving Dirichlet energies and the surface measure, we perform a local analysis of strictly convex portions of the boundary via second order shape derivatives. This allows in particular to exclude the presence of smooth regions with positive Gauss curvature in an optimal shape for Pólya-Szegö problem.
\end{abstract}

Mathematics Subject Classification. 49Q10, 31A15.

Received November 29, 2010.

Published online September 29, 2011.

\section{INTRODUCTION}

The initial motivation of this paper may be traced in a long-standing conjecture by Pólya and Szegö on minimal capacity sets. Precisely, the question is to find the convex sets in $\mathbb{R}^{3}$ of prescribed surface area which minimize the electrostatic capacity. In [35] it is conjectured that the minimizer is a planar disk, and the problem is currently open, though some advances in favor of the conjecture have been recently made in $[17,19]$. From the point of view of shape optimization, the most interesting feature is that the expected optimal shape is degenerate (the optimality of the disk among planar domains being straightforward). Hence one may reasonably expect that capacity enjoys a certain concavity-like property with respect to the shape variation.

Our first purpose is actually to investigate global concavity inequalities for functionals defined on the family of convex sets, in the perspective of applying them to solve shape optimization problems. The underlying idea is that minimizing a concave shape functional leads in a natural way to optimal sets which are either degenerate or, in a suitable sense, extremal. Of course, the notions of concavity and extremality become meaningful only after the family of convex sets has been endowed with a certain algebraic structure. The choice of such algebraic structure is a crucial step, which requires a careful balance between two opposite purposes: make the shape functional concave and the class of extremal convex bodies as restricted as possible.

Keywords and phrases. Convex bodies, concavity inequalities, optimization, shape derivatives, capacity.

${ }^{1}$ Laboratoire de Mathématiques UMR 5127, Université de Savoie, Campus Scientifique, 73376 Le-Bourget-du-Lac, France

2 Dipartimento di Matematica, Politecnico, Piazza Leonardo da Vinci, 32, 20133 Milano, Italy. ilaria.fragala@polimi.it

3 Ceremade UMR 7534, Université de Paris-Dauphine, Place du Maréchal De Lattre De Tassigny, 75775 Paris Cedex 16, France 
The algebraic structures most commonly considered on convex bodies are the Minkowski and Blaschke additions, which agree for planar sets but are deeply different in dimension $n \geq 3$. We refer the reader to Section 2 for their definitions, and to $[2,29,30]$ for their systematic use in solving isoperimetric-type problems (in a similar spirit, see also the recent papers [11], where global convexity properties associated to shadow systems allow to understand the extremality of parallelograms for the Mahler conjecture, and [32], where polyhedra appear as optimal shapes). The best choice among the Minkowski or Blaschke sum is self-evident in many situations, for instance in presence of a constraint which behaves linearly with respect to one of them: respectively, a prescribed mean width or surface area.

In the Minkowski structure, the queen mother among concavity inequalities is the Brunn-Minkowski theorem for the volume, a cornerstone in Convex Geometry dating back to one century ago, for which we refer to the extended survey paper [21]. A lot of efforts have been made during the years to show that the analogous concavity inequality holds true for several variational energies of Dirichlet type. A non-exhaustive list includes (functionals of) the first Dirichlet-eigenvalue of the Laplacian [6], the Newtonian capacity [4,10], the torsional rigidity [5], the $p$-capacity [15], the logarithmic capacity [13], the first eigenvalue of the Monge-Ampère operator [37], the Bernoulli constant [3]. For all these functionals, the Brunn-Minkowski inequality, combined with a result of Hadwiger, allows to identify balls as maximizers under prescribed mean width. Under the same constraint, minimizers must be searched among "extremal" sets in the Minkowski structure, usually called indecomposable bodies. Except in dimension $n=2$, when such bodies are reduced to (possibly degenerate) triangles, this is a very weak information since Minkowski-indecomposable sets are a dense family for $n \geq 3$.

On the contrary, in the Blaschke structure, a theorem of Bronshtein characterizes simplexes as the unique indecomposable bodies (in any dimension). For this reason, it is interesting to understand which kind of functionals enjoy a Blaschke-concavity property: their only possible minimizers under prescribed surface area will be either degenerate sets or simplexes. To the best of our knowledge, the unique known result in this direction is a theorem proved in the thirties by Kneser and Süss. It states a concavity inequality for the volume functional, which reads as the exact analogue of Brunn-Minkowski theorem in the Blaschke structure.

The starting point of this paper is actually the investigation of Kneser-Süss type inequalities for other functionals than volume, such as capacity and the first Dirichlet eigenvalue of the Laplacian. Quite surprisingly, we arrive fast at the conclusion that Blaschke-concavity inequalities for those functionals do not hold (see Props. 2.5 and 2.7). In particular we learn that, regarding concavity, capacity behaves differently from volume, though in the recent paper [22] the authors claim that it has the same "status" as volume, as it plays the role of its own dual set function in the dual Brunn-Minkowski theory. The argument we use is very simple, and relies on a Hadwiger-type theorem which allows to identify balls as maximizers of any Blaschke-concave functional under a surface area constraint (see Thm. 2.3). Incidentally, by the same method we provide a negative answer to the open question, stated for instance in [12], whether the second Dirichlet eigenvalue of the Laplacian satisfies a Brunn-Minkowksi type inequality (see Prop. 2.6).

Moving from these counterexamples, in order to gain concavity for functionals other than volume, we introduce a new algebraic structure on convex bodies. The reason why we drop the Blaschke addition is that it amounts to sum the surface area measures of two convex bodies, and the surface area measure is precisely related to the first variation of volume. In order to deal with a given functional different from volume, the natural idea is to sum its first variation measure at two given sets. Of course, some assumptions are needed to ensure the well-posedness of the notion (in the same way as Minkowski existence theorem must be invoked in the definition of Blaschke sum, see Defs. 3.1 and 3.2 for more details). However this can be done in good generality, and the abstract framework includes several significant examples, including capacity, torsional rigidity, and first Dirichlet eigenvalue of the Laplacian. With respect to this new sum, we prove that concavity holds true for any functional which satisfies the Brunn-Minkowski inequality and has first variation representable by a suitable integral formula: we believe that such result (Thm. 3.6) can be regarded as the natural analogue of Kneser-Süss theorem for the volume functional. We also show that our approach allows to deduce from the results in [18] a quantitative version of the Kneser-Süss inequality which involves the Fraenkel asymmetry (see Cor. 3.9). 
Next, still in the same algebraic structure, we are able to characterize indecomposable bodies in any dimension (see Thm. 3.13). We then turn attention to the resulting isoperimetric-type inequalities in shape optimization: some of them (like (3.12)) are rediscovered from the literature, some others (like (3.13)) are new, and some others (like (3.14)) rise delicate open questions. Unfortunately, the above described results do not help to solve the Pólya and Szegö conjecture, since the surface constraint is difficult to handle in the new algebraic structure.

Thus, with a change of perspective, we turn attention to investigate local properties of the optimal convex shapes by the analysis of the second order shape derivative. Contrary to the above discussed global concavity properties, in the local analysis of a strictly convex region we are allowed to use deformations which are not necessarily a resultant of the sum of convex sets. As usually, second order shape derivatives are highly technical and rather difficult to manage. Nevertheless, when computed at an optimal shape, they give an interesting information. Precisely, for a large class of functionals involving Dirichlet energies and the surface measure, and in particular for the Pólya-Szegö capacity problem, we prove that an optimal shape cannot have smooth open regions on its boundary with a non vanishing Gauss curvature (see Rem. 4.6). This can be done by observing that, otherwise, the second order shape derivative would not be a positive bilinear form with respect to a suitable class of shape deformations. We point out that the nonexistence of smooth regions with strictly positive curvatures, though of course does not prove entirely Pólya-Szegö conjecture, is the first qualitative information available on optimal sets: by now, the study of the problem had been carried out essentially by means of the first variation, which apparently does not contain enough information.

To conclude this introduction, we wish to mention two open questions which seem of relevant interest among those left by this paper: finding some methodology to exclude the optimality of polyhedra for the PólyaSzegö problem, and understanding whether a Blaschke-concavity inequality for capacity may hold true within some restricted class of convex bodies.

The contents are organized as follows.

In Section 2 we discuss concavity inequalities in the Minkowski and the Blaschke structure.

In Section 3 we define the new algebraic structure, and we give the related concavity and indecomposability results.

In Section 4 we deal with the local approach via second order shape derivatives.

\section{Global Minkowski and Blaschke-Concavity inequalities}

We start by introducing, in Sections 2.1 and 2.2 below, the Brunn-Minkowski and Kneser-Süss inequalities. This is done for convenience of the reader with a twofold aim: showing how these inequalities can be used in shape optimization problems, and paving the way to the counterexamples contained in Section 2.3.

Throughout the paper, we adopt the following notation: we let $\mathcal{K}^{n}$ be the class of convex bodies (compact convex sets) in $\mathbb{R}^{n}, \mathcal{K}_{0}^{n}$ be the class of convex bodies with nonempty interior, $\mathcal{B}^{n}$ be the class of balls, and $\Sigma^{n}$ be the class of simplexes.

By saying that a functional $F: \mathcal{K}^{n} \rightarrow \mathbb{R}^{+}$is $\alpha$-homogenous (for some $\alpha \neq 0$ ), we mean that $F(t K)=t^{\alpha} F(K)$ for every $K \in \mathcal{K}^{n}$ and every $t \in \mathbb{R}^{+}$.

For $K \in \mathcal{K}^{n}$, we recall that its support function $h(K)$ is defined on the unit sphere $\mathbb{S}^{n-1}$ of $\mathbb{R}^{n}$ by:

$$
h(K)(\nu):=\sup _{x \in K}(x \cdot \nu) \quad \forall \nu \in \mathbb{S}^{n-1} .
$$

Moreover, for $K \in \mathcal{K}_{0}^{n}$, we denote by $\nu_{K}: \partial K \rightarrow \mathbb{S}^{n-1}$ the Gauss map of $K$ (which is well defined $\mathcal{H}^{n-1}$-a.e. on $\partial K)$.

\subsection{Extremal problems under mean width constraint}

The Minkowski addition of two convex bodies $K$ and $L$ can be formally defined as the convex body $K+L$ such that

$$
h(K+L)=h(K)+h(L) .
$$


If a functional $F: \mathcal{K}^{n} \rightarrow \mathbb{R}^{+}$is Minkowski linear (meaning that $F(K+L)=F(K)+F(L)$ ), continuous in the Hausdorff distance and rigid motion invariant, then it is a constant multiple of the mean width $M(K)$ (see [39], p. 167). Recall that $M(K)$ is defined by

$$
M(K):=\frac{2}{\mathcal{H}^{n-1}\left(\mathbb{S}^{n-1}\right)} \int_{\mathbb{S}^{n-1}} h(K) \mathrm{d} \mathcal{H}^{n-1},
$$

and coincides with the perimeter of $K$ if we are in dimension $n=2$.

We say that a $\alpha$-homogenous functional $F: \mathcal{K}^{n} \rightarrow \mathbb{R}^{+}$satisfies the Brunn-Minkowski inequality if

$$
F^{1 / \alpha}(K+L) \geq F^{1 / \alpha}(K)+F^{1 / \alpha}(L) \quad \forall K, L \in \mathcal{K}^{n} .
$$

Besides volume (see [39], Thm. 6.1.1), several functionals satisfy the Brunn-Minkowski inequality, see the list of references given in the Introduction and also the survey paper [12]. To any of these functionals we may apply the following result. We give the proof for completeness since we were unable to find a precise reference in the literature (except for the sketch given in [3], Rem. 6.1); for similar results, see also the works [25,38].

Theorem 2.1. Assume that $F: \mathcal{K}^{n} \rightarrow \mathbb{R}^{+}$is $\alpha$-homogeneous, continuous in the Hausdorff distance, invariant under rigid motions, and satisfies the Brunn-Minkowski inequality. Consider the functional

$$
\mathcal{E}(K):=\frac{F^{1 / \alpha}(K)}{M(K)}
$$

Then:

- the maximum of $\mathcal{E}$ over $\mathcal{K}^{n}$ is attained on $\mathcal{B}^{n}$;

- if inequality (2.1) is strict for non-homothetic sets, then $\mathcal{E}$ attains its maximum over $\mathcal{K}^{n}$ only on $\mathcal{B}^{n}$; moreover, for $n=2, \mathcal{E}$ can attain its minimum over $\mathcal{K}^{2}$ only on $\Sigma^{2}$ (triangles) or on $\mathcal{K}^{2} \backslash \mathcal{K}_{0}^{2}$ (segments).

Proof. By a theorem of Hadwiger [39], Theorem 3.3.2, for any $K \in \mathcal{K}^{n}$ with affine hull of strictly positive dimension, there exists a sequence of Minkowski rotation means of $K$ which converges to a ball in the Hausdorff metric. Since $F$ satisfies (2.1) and the mean width is Minkowski linear, using also the continuity and invariance assumptions on $F$, it follows immediately that balls are maximizers for the quotient functional $\mathcal{E}$, and that no other maximizer exists if the strict inequality holds in (2.1) for non-homothetic sets. On the other hand, if such a strict inequality holds, the minimum of the shape functional $\mathcal{E}$ is attained necessarily on a Minkowskiindecomposable set. Indeed, if $K$ can be decomposed as $K=K^{\prime}+K^{\prime \prime}$, with $K^{\prime}$ and $K^{\prime \prime}$ non-homothetic, then $K$ cannot be a minimizer for $\mathcal{E}$, since

$$
\begin{aligned}
\mathcal{E}(K)=\frac{F^{1 / \alpha}\left(K^{\prime}+K^{\prime \prime}\right)}{M\left(K^{\prime}+K^{\prime \prime}\right)} & >\frac{F^{1 / \alpha}\left(K^{\prime}\right)+F^{1 / \alpha}\left(K^{\prime \prime}\right)}{M\left(K^{\prime}\right)+M\left(K^{\prime \prime}\right)} \\
& \geq \min _{i=1,2}\left\{\frac{F^{1 / \alpha}\left(K^{\prime}\right)}{M\left(K^{\prime}\right)}, \frac{F^{1 / \alpha}\left(K^{\prime \prime}\right)}{M\left(K^{\prime \prime}\right)}\right\} .
\end{aligned}
$$

Then the shape of possible minimizers for $\mathcal{E}$ over $\mathcal{K}^{n}$ can be deduced from the identification, holding in dimension $n=2$, of Minkowski indecomposable sets with (possibly degenerate) triangles [39], Theorem 3.2.11.

Remark 2.2. (i) If $n \geq 3$, the family of Minkowski indecomposable sets is dense in $\mathcal{K}^{n}$ [39], Theorem 3.2.14, so that arguing as above no qualitative information about minimizers of $\mathcal{E}$ can be extracted.

(ii) Clearly the shape functional $\mathcal{E}$ is invariant by dilations, and the problem of maximizing or minimizing $\mathcal{E}$ over $\mathcal{K}^{n}$ is equivalent to the problem of maximizing or minimizing $F$ under a prescribed mean width. A similar remark can be repeated for the shape optimization problems considered in the next sections.

Thanks to Theorem 2.1, we immediately get for instance that the maximum of the Newtonian capacity in $\mathcal{K}^{3}$, under the constraint of prescribed mean width, is attained at the ball. 


\subsection{Extremal problems under surface constraint}

Since in shape optimization problems, a surface area constraint occurs more frequently than a mean width one, it may be convenient to work with the Blaschke addition in place of the Minkowski one.

We recall that the surface area measure $\mu(K)$ of a convex body $K \in \mathcal{K}_{0}^{n}$ is the positive measure on $\mathbb{S}^{n-1}$ defined by

$$
\mu(K)[\omega]=\mathcal{H}^{n-1}\left(\nu_{K}^{-1}(\omega)\right) \quad \text { for all Borel sets } \omega \subseteq \mathbb{S}^{n-1} .
$$

For instance, if $K$ is a polyhedron with faces $F_{i}$ and normals with endpoints $P_{i}$, then $\mu(K)=\sum_{i} \mathcal{H}^{n-1}\left(F_{i}\right) \delta_{P_{i}}$, whereas if $K$ is smooth and has strictly positive curvatures, then $\mu(K)=\left(G_{K} \circ \nu_{K}^{-1}\right)^{-1} \mathcal{H}^{n-1}\left\llcorner\mathbb{S}^{n-1}\right.$, being $G_{K}$ the Gaussian curvature of $K$.

The Blaschke addition of two elements $K$ and $L$ of $\mathcal{K}_{0}^{n}$ is defined as the unique convex body $K \dot{+} L \in \mathcal{K}_{0}^{n}$ such that

$$
\mu(K+L)=\mu(K)+\mu(L) .
$$

(Accordingly, one may also define the Blaschke product $t \cdot K$ for any $t \in \mathbb{R}^{+}$and $K \in \mathcal{K}_{0}^{n}$ through the identity $\mu(t \cdot K)=t \mu(K)$, which amounts to say that $\left.t \cdot K=t^{1 /(n-1)} K\right)$.

About the correctness of this definition, we refer the reader to the comments in Section 3, Example 3.3.

If a functional $F: \mathcal{K}_{0}^{n} \rightarrow \mathbb{R}^{+}$is Blaschke linear (meaning that $F(K \dot{+} L)=F(K)+F(L)$ ), continuous in the Hausdorff distance and rigid motion invariant, then it is a constant multiple of the surface area $S(K)$ (see [24], Thm. 10.1).

By a slight abuse of language, and referring to the algebraic structure on the family of convex sets, we say that a $\alpha$-homogenous functional $F: \mathcal{K}^{n} \rightarrow \mathbb{R}^{+}$satisfies the Kneser-Süss inequality, if

$$
F^{(n-1) / \alpha}(K \dot{+} L) \geq F^{(n-1) / \alpha}(K)+F^{(n-1) / \alpha}(L) \quad \forall K, L \in \mathcal{K}_{0}^{n} .
$$

Theorem 2.3. Assume that $F: \mathcal{K}^{n} \rightarrow \mathbb{R}^{+}$is $\alpha$-homogeneous, continuous in the Hausdorff distance, invariant under rigid motions, and satisfies the Kneser-Süss inequality. Consider the functional

$$
\mathcal{E}(K):=\frac{F^{(n-1) / \alpha}(K)}{S(K)}
$$

Then:

- the maximum of $\mathcal{E}$ over $\mathcal{K}^{n}$ is attained on $\mathcal{B}^{n}$;

- if inequality (2.3) is strict for non-homothetic sets, then $\mathcal{E}$ attains its maximum over $\mathcal{K}^{n}$ only on $\mathcal{B}^{n}$; moreover, $\mathcal{E}$ can attain its minimum over $\mathcal{K}^{n}$ only on $\Sigma^{n}$ or on $\mathcal{K}^{n} \backslash \mathcal{K}_{0}^{n}$.

Proof. One can follow the same line as in the proof of Theorem 2.1. To obtain the maximality of balls, one has just to use, in place of Hadwiger theorem, an analogue result in the Blaschke structure [24], Corollary 9.3. To obtain information on possible minimizers, one has to apply the characterization of simplexes as the unique Blaschke-indecomposable bodies in $\mathcal{K}_{0}^{n}$ proved in [8], Theorem 1.

Remark 2.4. In dimension $n=2$, the statements of Theorems 2.1 and 2.3 coincide, because the Blaschke and Minkowski addition, respectively the perimeter and the mean width, agree.

As a straightforward consequence of Theorem 2.3, one gets for instance that:

- under the constraint of prescribed perimeter, the maximum and the minimum of logarithmic capacity in $\mathcal{K}^{2}$ are attained respectively at the ball and at a possibly degenerate triangle;

- under the constraint of prescribed perimeter, the maximum and the minimum of $p$-capacity in $\mathcal{K}^{2}$, with $p \in(1,2)$, are attained respectively at the ball and at a possibly degenerate triangle. 
Actually for the logarithmic capacity it is known that the solution is a degenerate triangle, i.e. a segment [36], p. 51 . Theorem 2.3 only says that the optimum is a possibly degenerate triangle. Further analysis is required to prove that the optimum is in fact a segment. For the $p$-capacity, this question seems to be open.

Contrary to the Brunn-Minkowski inequality, as far as we know, the only functional which is known to satisfy the Kneser-Süss inequality is the volume functional (see [39], Thm. 7.1.3). And in view of Theorem 2.3 it is of relevant interest to understand which functionals do satisfy the Kneser-Süss inequality. In particular, if the Newtonian capacity in $\mathbb{R}^{3}$ would satisfy the Kneser-Süss inequality, then the solution to the PólyaSzegö conjecture would get a step forward. Indeed, in that case the optimal could be only either a tetrahedron or a two dimensional body. Since the disk is known to be optimal among planar domains (see [17]), the analysis would be reduced to tetrahedrons, similarly as in the above mentioned minimization problem for the logarithimic capacity in two dimensions.

\subsection{Some non-concavity results}

We give below several negative results about the validity of the Kneser-Süss inequality (2.3) for variational functionals.

Proposition 2.5. In dimension $n=3$, the Newtonian capacity $\operatorname{Cap}(K)$ does not satisfy the the Kneser-Süss inequality (2.3).

Proof. If (2.3) would be true for $F(K)=\operatorname{Cap}(K)$, then by Theorem 2.1 the ball would have maximal capacity among convex bodies with a prescribed surface area. This contradicts the known fact that the quotient between the square of capacity and surface area diverges along a sequence of thinning prolate ellipsoids (see [17], Sect. 4).

Proposition 2.6. In dimension $n=2$, the second Dirichlet eigenvalue of the Laplacian, $\lambda_{2}(K)$, does not satisfy the Brunn-Minkowski inequality (2.1) (nor the Kneser-Süss inequality (2.3)).

Proof. If inequality (2.1) (or inequality (2.3)) would be true for $F(K)=\lambda_{2}(K)$, by Theorem 2.1 (or Thm. 2.3), the ball would have minimal $\lambda_{2}$ among planar convex bodies with a prescribed perimeter. This contradicts the geometric properties of minimizers for $\lambda_{2}$ under a perimeter constraint in two dimensions recently shown in [9], Theorem 2.5.

Proposition 2.7. In dimension $n=3$, the first Dirichlet eigenvalue of the Laplacian, $\lambda_{1}(K)$, does not satisfy the Kneser-Süss inequality (2.3).

Proof. In this case, if we try repeat the same argument used in the proof of Propositions 2.5 and 2.6, we do not arrive any longer to a contradiction, since the ball actually minimizes $\lambda_{1}$ among convex bodies with prescribed surface measure. Nevertheless, if one considers two parallelepipeds of the form

$$
P_{i}:=\left\{(x, y, z) \in \mathbb{R}^{3}: 0 \leq x \leq x_{i}, 0 \leq y \leq y_{i}, 0 \leq x \leq z_{i}\right\},
$$

the values of $\lambda_{1}\left(P_{1}\right), \lambda_{1}\left(P_{2}\right), \lambda_{1}\left(P_{1} \dot{+} P_{2}\right)$ can be computed explicitly. And it is a simple exercise to check that there are values of $x_{i}, y_{i}, z_{i}$ for which $(2.3)$ is false (e.g. $\left.x_{1}=z_{1}=z_{2}=1, x_{2}=4, y_{2} \rightarrow 0, y_{1} \rightarrow+\infty\right)$.

\section{Global Conchitity inequalities in A NeW Algebraic struCture}

The above counterexamples indicate that the Blaschke addition is not appropriate to gain concavity for functionals involving Dirichlet energies. This induced us to replace it by a new algebraic structure on convex bodies. The outcoming concavity and indecomposability results are proved in Section 3.1, and then some applications in shape optimization are discussed in Section 3.2. 


\subsection{The abstract results}

Denoting by $\mathcal{M}^{+}\left(\mathbb{S}^{n-1}\right)$ the class of positive measures on $\mathbb{S}^{n-1}$, we now introduce the class of maps $\mu$ : $\mathcal{K}_{0}^{n} \rightarrow \mathcal{M}^{+}\left(\mathbb{S}^{n-1}\right)$ which induce in a natural way an algebraic structure on $\mathcal{K}_{0}^{n}$.

Definition 3.1. We say that a map $\mu: \mathcal{K}_{0}^{n} \rightarrow \mathcal{M}^{+}\left(\mathbb{S}^{n-1}\right)$ is a parametrization of $\mathcal{K}_{0}^{n}$ if

(i) the image $\mu\left(\mathcal{K}_{0}^{n}\right)$ is a convex cone: $\nu_{1}, \nu_{2} \in \mu\left(\mathcal{K}_{0}^{n}\right), t_{1}, t_{2}>0 \Rightarrow t_{1} \nu_{1}+t_{2} \nu_{2} \in \mu\left(\mathcal{K}_{0}^{n}\right)$;

(ii) $\mu$ is injective up to translations: $\mu\left(K_{1}\right)=\mu\left(K_{2}\right) \Leftrightarrow K_{1}$ and $K_{2}$ are translates.

We say that $\mu$ is a $(\alpha-1)$-parametrization if, in addition,

(iii) $\mu$ is $(\alpha-1)$-homogeneous for some $\alpha \neq 1: K \in \mathcal{K}_{0}^{n}, t>0 \Rightarrow \mu(t K)=t^{\alpha-1} \mu(K)$.

For any parametrization of $\mathcal{K}_{0}^{n}$ according to the above definition, the following new operations on convex bodies are well-defined.

Definition 3.2. Let $\mu: \mathcal{K}_{0}^{n} \rightarrow \mathcal{M}^{+}\left(\mathbb{S}^{n-1}\right)$ be a parametrization of $\mathcal{K}_{0}^{n}$. For every couple of convex bodies $K, L \in \mathcal{K}_{0}^{n}$ and any $t>0$, we define $K{ }{ }_{\mu} L$ and $t \cdot{ }_{\mu} K$ as the convex bodies determined (up to a translation) by the equalities

$$
\mu\left(K+{ }_{\mu} L\right)=\mu(K)+\mu(L) \quad \text { and } \quad \mu\left(t \cdot{ }_{\mu} K\right)=t \mu(K) .
$$

By abuse of notation, we denote $K+{ }_{\mu} 0 \cdot{ }_{\mu} L:=K$. Several comments on these definitions are in order. We begin by giving some examples of parametrizations.

Example 3.3. Many $\alpha$-homogeneous functionals $F: \mathcal{K}_{0}^{n} \rightarrow \mathbb{R}^{+}$can be written in the integral form

$$
F(K)=\frac{1}{|\alpha|} \int h(K) \mathrm{d} \mu(K),
$$

being $\mu(\cdot)$ a $(\alpha-1)$-parametrization of $\mathcal{K}_{0}^{n}$. Here and in the sequel, when writing the expression at the right hand side of (3.1), we implicitly mean that $\alpha \neq 0$.

Some archetypal cases which fall in this framework are the following:

(i) The volume $\operatorname{Vol}(K)$ : in this case $\alpha=n$ and $\mu(K)$ is the surface area measure of $K$ defined in (2.2).

(ii) The 2-capacity $\operatorname{Cap}(K)$ : in this case $n \geq 3, \alpha=n-2$ and

$$
\mu(K)[\omega]=\int_{\nu_{K}^{-1}(\omega)}\left|\nabla u_{K}(x)\right|^{2} \mathrm{~d} \mathcal{H}^{n-1}(x) \quad \text { for all Borel sets } \omega \subseteq \mathbb{S}^{n-1},
$$

where $u_{K}$ is the electrostatic potential of $K$.

(iii) The first Dirichlet eigenvalue of Laplacian $\lambda_{1}(K)$ : in this case $\alpha=-2$ and $\mu(K)$ is given by (3.2), with $u_{K}$ equal to the first normalized eigenfunction of $K$.

(iv) The torsional rigidity $\tau(K)$ : in this case $\alpha=n+2$ and $\mu(K)$ is again written as in (3.2), begin now $u_{K}$ the solution to the equation $-\Delta u=1$ on $K$ with $u=0$ on $\partial K$.

For any of these functionals, the associated map $\mu$ turns out to be a parametrization of $\mathcal{K}_{0}^{n}$ since it takes values in the class $\mathcal{A}$ of so-called Alexandrov measures (positive measures on $\mathbb{S}^{n-1}$ which are not concentrated on any equator and have null barycenter), and satisfies the following Minkowski-type theorem: for every positive measure $\nu \in \mathcal{A}$ there exists a convex body $K \in \mathcal{K}_{0}^{n}$ (unique up to a translation) such that $\nu=\mu(K)$. In case of the volume, this was established by Minkowski for polyhedra and by Alexandrov in the general case [1]. The same result for capacity and for the first Dirichlet eigenvalue of the Laplacian is due to Jerison (see respectively [27], Thm. 0.8 and [28], Thm. 7.4), whereas for torsional rigidity it has been recently obtained by Colesanti and Fimiani [14], Theorems 1 and 2. Such results ensure that, for any of these maps $\mu$, we have $\mu\left(\mathcal{K}_{0}^{n}\right)=\mathcal{A}$, which is a convex cone, and injectivity holds up to translations. Thus (i) and (ii) in Definition 3.1 are satisfied. We remark that also condition (iii) of the same definition is fulfilled (except for $\operatorname{Vol}(K)$ in dimension $n=1$, and $\operatorname{Cap}(K)$ in dimension $n=3)$. 
Remark 3.4. (i) If $\mu$ is a $(\alpha-1)$-parametrization, by homogeneity we have $t \cdot{ }_{\mu} K:=t^{1 /(\alpha-1)} K$.

(ii) When $\mu$ is the surface area measure, Definition 3.2 gives exactly the Blasckhe structure. In spite, as pointed out in [23], area measures of intermediate order cannot be used in order to define an addition of convex bodies.

(iii) For any of the parametrizations appearing in Example 3.3, the $\mu$-addition of two convex bodies provides a result geometrically different from their Minkowski addition. For instance, in $\mathbb{R}^{3}$ one can take two regular tetrahedra, one of which is obtained by the other through a rotation of $\pi / 2$, such that their Minkowski sum has 14 faces ( $c f$. [2]); clearly their $\mu$-sum, for each of the parametrizations $\mu$ in Example 3.3, has just 8 faces, whose areas depend on the choice of $\mu$.

Relying on the algebraic structure introduced in Definition 3.2, a new natural notion of concavity emerges for the associated integral functionals.

Definition 3.5. Let $\mu$ be a $(\alpha-1)$-parametrization of $\mathcal{K}_{0}^{n}$, and let $F: \mathcal{K}_{0}^{n} \rightarrow \mathbb{R}^{+}$be given by (3.1). We say that $F$ is $\mu$-concave if

$$
F^{1-(1 / \alpha)}\left(K+{ }_{\mu} L\right) \geq F^{1-(1 / \alpha)}(K)+F^{1-(1 / \alpha)}(L) \quad \forall K, L \in \mathcal{K}_{0}^{n} .
$$

We are going to show that this concavity property is strictly related with the Brunn-Minkowski inequality. In particular, it holds true for any of the functionals considered in Example 3.3, since they satisfy the assumptions of Theorem 3.6 below (a comprehensive reference for this claim is [12]). Thus, we may affirm that (3.3) it is the natural extension of the Kneser-Süss inequality for the volume functional.

Theorem 3.6. Let $\mu$ be $a(\alpha-1)$-parametrization $\mu$ of $\mathcal{K}_{0}^{n}$, and let $F: \mathcal{K}_{0}^{n} \rightarrow \mathbb{R}^{+}$be given by (3.1). Assume that F satisfies the Brunn-Minkowski inequality (2.1) and that its first variation under Minkowski sums can be written as

$$
\frac{\mathrm{d}}{\mathrm{d} t} F(K+t L)_{\mid t=0+}=\frac{\alpha}{|\alpha|} \int h(L) \mathrm{d} \mu(K) .
$$

Then $F$ is $\mu$-concave. Moreover, if equality occurs in (2.1) only when $K$ and $L$ are homothetic, then the same property holds for (3.3).

Remark 3.7. Notice that, if $F: \mathcal{K}_{0}^{n} \rightarrow \mathbb{R}^{+}$is a $\alpha$-homogeneous functional which satisfies (3.4), it follows automatically that it can be written in the integral form (3.1). This means that only assumption (3.4) is crucial. We emphasize that such assumption is less restrictive than it may appear since (3.4) is nothing else than a representation formula for the shape derivative. Indeed, if the functional $F$ is shape derivable, under mild assumptions the Hadamard structure theorem for the first shape derivative applies. Consequently, for any deformation by a vector field $V \in C_{0}^{\infty}\left(\mathbb{R}^{n} ; \mathbb{R}^{n}\right)$, the first shape derivative is a linear form depending on the normal component of $V$ on $\partial K$. Moreover, under continuity assumptions, the shape gradient can be identified with a function $g_{K} \in L^{1}(\partial K)$, so that

$$
\frac{\mathrm{d}}{\mathrm{d} t} F((I d+t V)(K))_{\left.\right|_{t=0^{+}}}=\int_{\partial K} g_{K}\left(V \cdot \nu_{K}\right) \mathrm{d} \mathcal{H}^{n-1} .
$$

Though the deformation through the Minkowski addition is in general not induced by a vector field, a similar formal analysis leads to representing the derivative in the left hand side of (3.4) as

$$
\frac{\mathrm{d}}{\mathrm{d} t} F(K+t L)_{\left.\right|_{t=0^{+}}}=\int_{\mathbb{S}^{n-1}} \tilde{g}_{K} h(L) \mathrm{d} \mathcal{H}^{n-1} .
$$

Thus, setting $\mu(K):=|\alpha| \tilde{g}_{K} \mathcal{H}^{n-1}\left\llcorner\mathbb{S}^{n-1}\right.$, we find a map $K \mapsto \mu(K)$ which allows to represent $F$ in the integral form (3.1). Of course, establishing whether $\mu$ is in fact a parametrization according to Definition 3.1 is not a trivial question. 
Proof of Theorem 3.6. By using (3.1) and (3.4), we may write:

$$
\begin{aligned}
F\left(K+{ }_{\mu} L\right) & =\frac{1}{|\alpha|} \int h\left(K+{ }_{\mu} L\right) \mathrm{d} \mu\left(K+{ }_{\mu} L\right) \\
& =\frac{1}{|\alpha|} \int h\left(K+{ }_{\mu} L\right) \mathrm{d} \mu(K)+\frac{1}{|\alpha|} \int h\left(K+{ }_{\mu} L\right) \mathrm{d} \mu(L) \\
& =\frac{1}{\alpha} \frac{\mathrm{d}}{\mathrm{d} t} F\left(K+t\left(K+{ }_{\mu} L\right)\right)_{\left.\right|_{t=0^{+}}}+\frac{1}{\alpha} \frac{\mathrm{d}}{\mathrm{d} t} F\left(L+t\left(K+{ }_{\mu} L\right)\right)_{\left.\right|_{t=0^{+}}} .
\end{aligned}
$$

Since by assumption $F$ satisfies the Brunn Minkowski inequality (2.1), for all $C$ and $C^{\prime}$ in $\mathcal{K}^{n}$ the function

$$
f(t):=F^{1 / \alpha}\left(C+t C^{\prime}\right)-F^{1 / \alpha}(C)-t F^{1 / \alpha}\left(C^{\prime}\right)
$$

is nonnegative for $t \geq 0$. Since $f(0)=0$, this means that $f^{\prime}(0) \geq 0$, which gives

$$
\frac{1}{\alpha} \frac{\mathrm{d}}{\mathrm{d} t} F\left(C+t C^{\prime}\right)_{\left.\right|_{t=0^{+}}} \geq F^{1-(1 / \alpha)}(C) F^{1 / \alpha}\left(C^{\prime}\right)
$$

Applying this inequality once with $C=K$ and $C^{\prime}=K+{ }_{\mu} L$, and once with $C=L$ and $C^{\prime}=K+{ }_{\mu} L$, we infer

$$
F\left(K+{ }_{\mu} L\right) \geq\left[F^{1-(1 / \alpha)}(K)+F^{1-(1 / \alpha)}(L)\right] F^{1 / \alpha}\left(K+{ }_{\mu} L\right),
$$

and inequality (3.3) follows dividing by $F^{1 / \alpha}\left(K+{ }_{\mu} L\right)$.

Assume now that (3.3) holds with equality sign. By the above proof it follows that, taking $C=K$ and $C^{\prime}=K+{ }_{\mu} L$, the function $f$ defined as in (3.5) satisfies $f^{\prime}(0)=0$. But, by the Brunn Minkowksi inequality (2.1), we know that $f$ is both nonnegative and concave. Hence it must vanish identically. By assumption, this implies that $K$ and $K+{ }_{\mu} L$ are homothetic, and hence $K$ and $L$ are homothetic.

Under the same assumptions of Theorem 3.6, following the same lines one can prove the following refined concavity inequality. Let

$$
\sigma(K, L):=\max \left\{\frac{F(K)}{F(L)}, \frac{F(L)}{F(K)}\right\},
$$

and $R: \mathcal{K}_{0}^{n} \times \mathcal{K}_{0}^{n} \rightarrow \mathbb{R}^{+}$be an "asymmetry distance", i.e.

(i) $\forall K, L \in \mathcal{K}_{0}^{n}, \quad \forall t, s>0, \quad R(t K, s L)=R(K, L)$,

(ii) $\exists c>0, \quad \forall K, L, S \in \mathcal{K}_{0}^{n}, \quad R(K, L)+R(L, S) \geq c R(K, S)$.

Proposition 3.8. Under the same assumptions of Theorem 3.6, if the Brunn-Minkowski inequality (2.1) holds in the quantitative form

$$
F^{1 / \alpha}(K+L) \geq\left(F^{1 / \alpha}(K)+F^{1 / \alpha}(L)\right)\left(1+\frac{R(K, L)}{\sigma(K, L)^{1 / \alpha}}\right) \quad \forall K, L \in \mathcal{K}_{0}^{n},
$$

then (3.3) holds in the quantitative form

$$
F^{1-(1 / \alpha)}\left(K+{ }_{\mu} L\right) \geq\left(F^{1-(1 / \alpha)}(K)+F^{1-(1 / \alpha)}(L)\right)\left(1+\frac{c}{2} \frac{R(K, L)}{\sigma(K, L)^{1-1 / \alpha}}\right) \quad \forall K, L \in \mathcal{K}_{0}^{n} .
$$

In particular, for $F(K)=\operatorname{Vol}(K)$, we obtain a quantitative version of the Kneser-Süss inequality. Let us denote for every $K, L \in \mathcal{K}_{0}^{n}$ the Fraenkel relative asymmetry

$$
A(K, L):=\inf _{x_{0} \in \mathbb{R}^{n}}\left\{\frac{\operatorname{Vol}\left(K \Delta\left(x_{0}+\lambda L\right)\right)}{\operatorname{Vol}(K)}\right\}, \quad \text { where } \lambda:=\left(\frac{\operatorname{Vol}(K)}{\operatorname{Vol}(L)}\right)^{1 / n} .
$$


We notice that, for every $p \geq 1, A(\cdot, \cdot)^{p}$ is an asymmetry distance which satisfies (i) and (ii) above.

Corollary 3.9. There exists a constant $c_{n}>0$ depending only on the space dimension such that

$$
\operatorname{Vol}(K \dot{+} L)^{1-(1 / n)} \geq\left(\operatorname{Vol}(K)^{1-(1 / n)}+\operatorname{Vol}(L)^{1-(1 / n)}\right)\left(1+\frac{A(K, L)^{2}}{c_{n} \sigma(K, L)^{1-(1 / n)}}\right) \quad \forall K, L \in \mathcal{K}_{0}^{n} .
$$

Proof. The proof is a direct consequence of the previous proposition and of the quantitative Brunn-Minkowski inequality proved in [18].

As a consequence of Theorem 3.6, functionals as in the statement are monotone with respect to the associated parametrization.

Corollary 3.10. Let $F$ satisfy the assumptions of Theorem 3.6. If $K, L \in \mathcal{K}_{0}^{n}$ are such that $\mu(K) \leq \mu(L)($ as measures on $\left.\mathbb{S}^{n-1}\right)$, then $F(K) \leq F(L)$.

Proof. We argue as in the proof of [2], Theorem 7.1. For $t \in(0,1)$, set $\mu_{t}:=\mu(L)-t \mu(K)$. Since by assumption $\mu(L) \geq \mu(K)$, and since $\mu$ is a parametrization of $\mathcal{K}_{0}^{n}$, there exists a convex body $M_{t}$ (unique up to a translation), such that $\mu_{t}=\mu\left(M_{t}\right)$. Then we have $\mu(L)=\mu\left(M_{t}\right)+t \mu(K)$, which implies $L=M_{t}+{ }_{\mu} t \cdot{ }_{\mu} K$. Then (3.3) implies

$$
F^{1-(1 / \alpha)}(L)=F^{1-(1 / \alpha)}\left(M_{t}+{ }_{\mu} t \cdot{ }_{\mu} K\right) \geq F^{1-(1 / \alpha)}\left(M_{t}\right)+t F^{1-(1 / \alpha)}(K) \geq t F^{1-(1 / \alpha)}(K),
$$

and the thesis follows by letting $t$ tend to 1 .

With a proof similar to the one of Theorem 3.6, we obtain the following "dual" statement.

Theorem 3.11. Let $\mu$ be a $(\alpha-1)$-parametrization of $\mathcal{K}_{0}^{n}$, and let $F: \mathcal{K}_{0}^{n} \rightarrow \mathbb{R}^{+}$be given by (3.1). Assume that $F$ is $\mu$-concave and that its first variation under $\mu$-sums can be written as

$$
\frac{\mathrm{d}}{\mathrm{d} t} F\left(L+{ }_{\mu} t \cdot{ }_{\mu} K\right)_{\left.\right|_{t=0^{+}}}=\frac{\alpha}{|\alpha|} \frac{1}{\alpha-1} \int h(L) \mathrm{d} \mu(K) .
$$

Then F satisfies the Brunn Minkowski inequality (2.1). Moreover, if equality occurs in (3.3) only when $K$ and $L$ are homothetic, the same property holds for (2.1).

Proof. By using (3.1) and (3.8), we have:

$$
\begin{aligned}
F(K+L) & =\frac{1}{|\alpha|} \int h(K+L) \mathrm{d} \mu(K+L) \\
& =\frac{1}{|\alpha|} \int h(K) \mathrm{d} \mu(K+L)+\frac{1}{|\alpha|} \int h(L) \mathrm{d} \mu(K+L) \\
& =\frac{\alpha-1}{\alpha} \frac{\mathrm{d}}{\mathrm{d} t} F\left(K+{ }_{\mu} t \cdot{ }_{\mu}(K+L)\right)_{\left.\right|_{t=0^{+}}}+\frac{\alpha-1}{\alpha} \frac{\mathrm{d}}{\mathrm{d} t} F\left(L+{ }_{\mu} t \cdot{ }_{\mu}(K+L)\right)_{\left.\right|_{t=0+}}
\end{aligned}
$$

Since now $F$ satisfies (3.3), for all $C$ and $C^{\prime}$ in $\mathcal{K}^{n}$ the function

$$
f(t):=F^{1-(1 / \alpha)}\left(C+{ }_{\mu} t \cdot{ }_{\mu} C^{\prime}\right)-F^{1-(1 / \alpha)}(C)-t F^{1-(1 / \alpha)}\left(C^{\prime}\right)
$$

is nonnegative for $t \geq 0$. Since $f(0)=0$, this implies $f^{\prime}(0) \geq 0$, and hence

$$
\frac{\alpha-1}{\alpha} \frac{\mathrm{d}}{\mathrm{d} t} F\left(C+{ }_{\mu} t \cdot{ }_{\mu} C^{\prime}\right)_{\left.\right|_{t=0^{+}}} \geq F^{1 / \alpha}(C) F^{1-(1 / \alpha)}\left(C^{\prime}\right) .
$$


Applying this inequality once with $C=K$ and $C^{\prime}=K+L$, and once with $C=L$ and $C^{\prime}=K+L$, we infer

$$
F(K+L) \geq\left[F^{1 / \alpha}(K)+F^{(1 / \alpha)}(L)\right] F^{1-(1 / \alpha)}(K+L),
$$

and (2.1) follows dividing by $F^{1-(1 / \alpha)}(K+L)$.

If (2.1) holds with equality sign, the function $f$ defined as in (3.9), with $C=K$ and $C^{\prime}=K+L$, satisfies $f^{\prime}(0)=0$. Since by (3.3) $f$ is both nonnegative and concave, it vanishes identically. By assumption, this implies that $K$ and $K+L$ are homothetic, and hence $K$ and $L$ are homothetic.

In view of applications of Theorem 3.6 to extremal problems, it is useful to give the following

Definition 3.12. Let $\mu$ be a parametrization of $\mathcal{K}_{0}^{n}$. By saying that $K \in \mathcal{K}_{0}^{n}$ is $\mu$-indecomposable, we mean that the equality $K=K^{\prime}+{ }_{\mu} K^{\prime \prime}$ implies that $K^{\prime}$ and $K^{\prime \prime}$ are $\mu$-scalar multiples of $K$.

When $\mu$ is the surface area measure, a theorem of Bronshtein [8], Theorem 1 characterizes simplexes as the unique Blaschke-indecomposable bodies. The same proof allows to obtain the following more general statement. We denote by $\mathcal{A}$ the class of Alexandrov measures on $\mathbb{S}^{n-1}$ (defined as in Ex. 3.3).

Theorem 3.13. Let $\mu$ be a parametrization of $\mathcal{K}_{0}^{n}$, with $\mu\left(\mathcal{K}_{0}^{n}\right)=\mathcal{A}$. Then a convex body $K \in \mathcal{K}_{0}^{n}$ is $\mu$-indecomposable if and only if $\operatorname{spt}(\mu(K))$ consists exactly of $(n+1)$ distinct points in $\mathbb{S}^{n-1}$.

Remark 3.14. For any of the parametrizations in Example 3.3, since $\operatorname{spt}(\mu(K))$ coincides with the image of the Gauss map $\nu_{K}$, Theorem 3.13 tells that the unique $\mu$-indecomposable bodies are simplexes, exactly as it happens for the Blaschke sum.

Proof of Theorem 3.13. (i) Let us show that, if $\operatorname{spt}(\mu(K))$ contains $(n+2)$ distinct points $\left\{\xi_{1}, \ldots, \xi_{n+2}\right\}$ in $\mathbb{S}^{n-1}$, then $K$ is not $\mu$-indecomposable.

For every $i \in\{1, \ldots, n+2\}$, let $\omega_{i}$ be pairwise disjoint neighbourhoods of $\xi_{i}$ in $\mathbb{S}^{n-1}$, and let $C$ be the complement of their union in $\mathbb{S}^{n-1}$. Since $\mu(K)$ has null baricenter, we have

$$
\sum_{i=1}^{n+2} \int_{\omega_{i}} x \mathrm{~d} \mu(K)+\int_{C} x \mathrm{~d} \mu(K)=0 .
$$

For generic positive coefficients $\gamma_{i}$ (to be chosen later), consider the measure

$$
\nu:=\sum_{i=1}^{n+2} \gamma_{i} \mu\left\llcorner\omega_{i}+\frac{1}{2} \mu\llcorner C .\right.
$$

It is clear that, for any choice of $\gamma_{i}>0, \nu$ is not concentrated on any equator of $\mathbb{S}^{n-1}$. If we impose that $\nu$ has null barycenter, we obtain the following system of $n$ equations in the $n+2$ unknown $\left(\gamma_{1}, \ldots, \gamma_{n+2}\right)$ :

$$
\sum_{i=1}^{n+2} \gamma_{i} \int_{\omega_{i}} x \mathrm{~d} \mu(K)+\frac{1}{2} \int_{C} x \mathrm{~d} \mu(K)=0 .
$$

Since by (3.10) we know that a solution exists (by taking $\gamma_{i}=1 / 2$ for all $i$ ), we infer that there exists a 2-dimensional linear subspace $V$ of $\mathbb{R}^{n+2}$ such that any $\gamma \in(1 / 2, \ldots, 1 / 2)+V$ is a solution. Therefore, we may choose solutions $\left(\gamma_{1}^{\prime}, \ldots, \gamma_{n+2}^{\prime}\right)$ and $\left(\gamma_{1}^{\prime \prime}, \ldots, \gamma_{n+2}^{\prime \prime}\right)$ such that $\gamma_{i}^{\prime}$ are not all equal, $\gamma_{i}^{\prime}>0, \gamma_{i}^{\prime \prime}>0$, and $\gamma_{i}^{\prime}+\gamma_{i}^{\prime \prime}=1$.

We set $\nu^{\prime}$ and $\nu^{\prime \prime}$ the measures defined as in (3.11), with $\gamma_{i}$ equal to $\gamma_{i}^{\prime}$ and $\gamma_{i}^{\prime \prime}$ respectively. Since $\nu^{\prime}$ and $\nu^{\prime \prime}$ belong to $\mathcal{A}$, and by assumption $\mu$ is a parametrization of $\mathcal{K}_{0}^{n}$ with $\mu\left(\mathcal{K}_{0}^{n}\right)=\mathcal{A}$, there exist $K^{\prime}$ and $K^{\prime \prime}$ in $\mathcal{K}_{0}^{n}$ such that $\mu\left(K^{\prime}\right)=\nu^{\prime}$ and $\mu\left(K^{\prime \prime}\right)=\nu^{\prime \prime}$. By construction, we have $\mu(K)=\mu\left(K^{\prime}\right)+\mu\left(K^{\prime \prime}\right)$, and $\mu\left(K^{\prime}\right)$ is not a multiple of $\mu(K)$. Then $K$ is not $\mu$-indecomposable. 
On the other hand, if $\operatorname{spt}(\mu(K))$ contains strictly less than $n+1$ points in $\mathbb{S}^{n-1}$, then $\mu(K)$ cannot belong to $\mathcal{A}$. Indeed, the null barycenter condition implies that these points are linearly dependent, hence $\mu$ is concentrated on the intersection of some hyperplane with $\mathbb{S}^{n-1}$.

Therefore we have proved that, if $K$ is $\mu$-indecomposable, necessarily $\operatorname{spt}(\mu(K))$ is made exactly by $(n+1)$ distinct points in $\mathbb{S}^{n-1}$.

(ii) Viceversa, assume that $\operatorname{spt}(\mu(K))=\left\{\xi_{1}, \ldots, \xi_{n+1}\right\}$, and let us show that $K$ is $\mu$-indecomposable. Assume that $K=K^{\prime}+{ }_{\mu} K^{\prime \prime}$. The equality $\mu(K)=\mu\left(K^{\prime}\right)+\mu\left(K^{\prime \prime}\right)$ implies that $\operatorname{spt}\left(\mu\left(K^{\prime}\right)\right)$ and $\operatorname{spt}\left(\mu\left(K^{\prime \prime}\right)\right)$ are contained into $\left\{\xi_{1}, \ldots, \xi_{n+1}\right\}$. For $i=1, \ldots, n+1$, let $\sigma_{i}$ and $\lambda_{i}$ be positive numbers such that

$$
\mu(K)\left[\xi_{i}\right]=\sigma_{i} \quad \text { and } \quad \mu\left(K^{\prime}\right)\left[\xi_{i}\right]=\lambda_{i} \sigma_{i} .
$$

Since both $\mu(K)$ and $\mu\left(K^{\prime}\right)$ have null barycenter, we have

$$
\lambda_{1} \sum_{i=1}^{n+1} \sigma_{i} \xi_{i}=0 \quad \text { and } \quad \sum_{i=1}^{n+1} \lambda_{i} \sigma_{i} \xi_{i}=0 .
$$

By subtraction, we obtain

$$
\sum_{i=2}^{n+1}\left(\lambda_{i}-\lambda_{1}\right) \sigma_{i} \xi_{i}=0
$$

If $\lambda_{i} \neq \lambda_{1}$ for some $i \in\{2, \ldots, n+1\}$, then $\left\{\xi_{2}, \ldots \xi_{n+2}\right\}$ would be linearly dependent, so that $\mu(K)$ would be concentrated on the intersection of $\mathbb{S}^{n-1}$ with an hyperplane through the origin, against the assumption that $\mu(K) \in \mathcal{A}$. Therefore it must be $\lambda_{i}=\lambda_{1}$ for all $i \in\{2, \ldots, n+1\}$, which means that $K^{\prime}$ equals $\lambda_{1} \cdot{ }_{\mu} K$.

\subsection{Isoperimetric-like problems}

We now focus attention on the isoperimetric-like problems of maximizing or minimizing the dilation invariant quotient

$$
\mathcal{E}(K):=\frac{F^{1-(1 / \alpha)}(K)}{\int \mathrm{d} \mu(K)},
$$

being $F$ a $\alpha$-homogeneous functional which can be written under the integral form (3.1).

Theorem 3.15. Let $F: \mathcal{K}_{0}^{n} \rightarrow \mathbb{R}^{+}$be given by (3.1) for some $(\alpha-1)$-homogeneous map $\mu: \mathcal{K}_{0}^{n} \rightarrow \mathcal{M}^{+}\left(\mathbb{S}^{n-1}\right)$. Then:

- if F satisfies the Brunn-Minkowski inequality (2.1), then the maximum of $\mathcal{E}$ over $\mathcal{K}_{0}^{n}$ is attained on $\mathcal{B}^{n}$;

- if $\mu$ is a parametrization of $\mathcal{K}_{0}^{n}$ and $F$ satisfies (3.3), with strict inequality for non-homothetic sets, then $\mathcal{E}$ can attain its minimum over $\mathcal{K}_{0}^{n}$ only at a $\mu$-indecomposable set.

Proof. Assume that $F$ satisfies (2.1). For a fixed $K \in \mathcal{K}_{0}^{n}$, let us denote by $B$ the ball in $\mathcal{K}_{0}^{n}$ with $\int \mathrm{d} \mu(B)=$ $\int \mathrm{d} \mu(K)$. We use once more the argument that the function

$$
f(t):=F^{1 / \alpha}(K+t B)-F^{1 / \alpha}(K)-t F^{1 / \alpha}(B)
$$

is nonnegative for $t \geq 0$ to deduce that $f^{\prime}(0) \geq 0$, which gives

$$
\frac{1}{|\alpha|} h(B) \int \mathrm{d} \mu(K)=\frac{1}{\alpha} \frac{\mathrm{d}}{\mathrm{d} t} F(K+t B)_{\left.\right|_{t=0^{+}}} \geq F^{1-(1 / \alpha)}(K) F^{1 / \alpha}(B) .
$$

Since $|\alpha|=(F(B))^{-1} h(B) \int \mathrm{d} \mu(B)$, the above inequality yields $F(B) \geq F(K)$. 
Assume now that $\mu$ is a parametrization of $\mathcal{K}_{0}^{n}$ and that $F$ satisfies (3.3), with strict inequality for nonhomothetic sets. If $K$ can be decomposed as $K=K^{\prime}+{ }_{\mu} K^{\prime \prime}$, with $K^{\prime}$ and $K^{\prime \prime}$ non-homotetic, then by arguing as in the proof of Theorem 2.1 one obtains that $K$ cannot be a minimizer for $\mathcal{E}$.

As a consequence of Theorems 3.6, 3.13, and 3.15, we deduce

Corollary 3.16. For any of the functionals in Example 3.3, the unique maximizers of $\mathcal{E}$ over $\mathcal{K}_{0}^{n}$ are balls, and any minimizer of $\mathcal{E}$ over $\mathcal{K}_{0}^{n}$ (if it exists) is a simplex.

Let us examine more in detail each case.

- Volume. When $F(K)=\operatorname{Vol}(K)$, the inequality $\mathcal{E}(K) \leq \mathcal{E}(B)$ corresponds to the classical isoperimetric inequality for convex bodies, whereas clearly $\inf _{\mathcal{K}_{0}^{n}} \mathcal{E}=0$.

- Capacity. When $F(K)=\operatorname{Cap}(K)(n \geq 3)$, the inequality $\mathcal{E}(K) \leq \mathcal{E}(B)$ corresponds to

$$
\operatorname{Cap}(K)^{(n-3) /(n-2)} \leq c_{n} \int_{\partial K}\left|\nabla u_{K}\right|^{2} \mathrm{~d} \mathcal{H}^{n-1} \quad \forall K \in \mathcal{K}_{0}^{n},
$$

where $u_{K}$ is the electrostatic potential of $K$ and the dimensional constant $c_{n}$ is chosen so that equality holds when $K$ is a ball. Inequality (3.12) was already known: it has been proved by Jerison in [27], Corollary 3.19. On the other hand, Lemma 4.13 in the same paper implies that $\mathcal{E}\left(K_{h}\right)$ is infinitesimal for any sequence $\left\{K_{h}\right\}$ of convex bodies which converges to a convex set $K_{0}$ contained into a $(n-1)$-hyperplane. Therefore, we have again $\inf _{\mathcal{K}_{0}^{n}} \mathcal{E}=0$.

- Torsional rigidity. When $F(K)=\tau(K)$, the inequality $\mathcal{E}(K) \leq \mathcal{E}(B)$ corresponds to

$$
\tau(K)^{(n+1) /(n+2)} \leq c_{n} \int_{\partial K}\left|\nabla u_{K}\right|^{2} \mathrm{~d} \mathcal{H}^{n-1} \quad \forall K \in \mathcal{K}_{0}^{n},
$$

where now $u_{K}$ is the solution to the equation $-\Delta u=1$ on $K$ with $u=0$ on $\partial K$, and $c_{n}$ is chosen so that equality holds when $K$ is a ball.

To the best of our knowledge, the isoperimetric-like inequality (3.13) is new. In view of the first variation formula (3.4), it can be rephrased as follows: among convex domains with prescribed torsional rigidity, the ball is the most stable when perturbed by Minkowski addition of a ball.

On the other hand, we claim that the infimum is again zero. Indeed (for $n=2$ ), let $R_{l}$ denote the rectangle $[0, l] \times[0,1]$. Then the unique solution $u_{l}$ in $H_{0}^{1}\left(R_{l}\right)$ to the equation $-\Delta u=1$ in $R_{l}$ can be explicitly determined as (see for instance [16])

$$
u_{l}(x, y)=\frac{l x-x^{2}}{2}-\frac{4 l^{2}}{\pi^{3}} \sum_{k=0}^{\infty} \frac{\sin [(2 k+1) \pi x]}{(2 k+1)^{3}\left(\mathrm{e}^{(2 k+1) \pi / l}+1\right)}\left\{\mathrm{e}^{(2 k+1) \pi y / l}+\mathrm{e}^{(2 k+1) \pi(1-y) / l}\right\} .
$$

From this formula one can easily check that, as $l \rightarrow 0$,

$$
\tau\left(R_{l}\right)=O\left(l^{3}\right) \quad \text { and } \quad \int_{\partial R_{l}}\left|\nabla u_{l}\right|^{2} \mathrm{~d} \mathcal{H}^{1}=O\left(l^{2}\right),
$$

so that

$$
\lim _{l \rightarrow 0} \frac{\tau\left(R_{l}\right)^{3 / 4}}{\int_{\partial R_{l}}\left|\nabla u_{l}\right|^{2} \mathrm{~d} \mathcal{H}^{1}}=0
$$


- The first Dirichlet eigenvalue. When $F(K)=\lambda_{1}(K)$, the inequality $\mathcal{E}(K) \leq \mathcal{E}(B)$ reads

$$
\lambda_{1}(K)^{3 / 2} \leq c_{n} \int_{\partial K}\left|\nabla u_{K}\right|^{2} \mathrm{~d} \mathcal{H}^{n-1} \quad \forall K \in \mathcal{K}_{0}^{n},
$$

where $u_{K}$ is the first Dirichlet eigenfunction of $K$ and the dimensional constant $c_{n}$ is chosen so that equality holds when $K$ is a ball.

Also this result seems to be new and means: among convex domains with prescribed first Dirichlet Laplacian eigenvalue, the ball is the most stable when perturbed by Minkowski addition of a ball.

In this case the analysis of thinning rectangles leads to guess that the infimum

$$
\inf _{K \in \mathcal{K}_{0}^{2}} \frac{\lambda_{1}(K)^{3 / 2}}{\int_{\partial K}\left|\nabla u_{K}\right|^{2} \mathrm{~d} \mathcal{H}^{1}}
$$

remains strictly positive. Indeed let $R_{l}$ denote as above the rectangle $[0, l] \times[0,1]$ in $\mathbb{R}^{2}$, and let now $u_{l}$ be the first Dirichlet eigenfunction of the Laplacian on $R_{l}$. Then

$$
u_{l}(x, y)=\frac{2}{\sqrt{l}} \sin \left(\frac{\pi x}{l}\right) \sin (\pi y) \quad \text { and } \quad \lambda_{1}\left(R_{l}\right)=\pi^{2}\left(\frac{1}{l^{2}}+1\right) .
$$

By direct computation one gets

$$
\int_{\partial R_{l}}\left|\nabla u_{l}\right|^{2} \mathrm{~d} \mathcal{H}^{1}=4 \pi\left(\frac{1}{l^{3}}+1\right)
$$

so that

$$
\lim _{l \rightarrow 0} \frac{\lambda_{1}\left(R_{l}\right)^{3 / 2}}{\int_{\partial R_{l}}\left|\nabla u_{l}\right|^{2} \mathrm{~d} \mathcal{H}^{1}}=\frac{\pi^{2}}{4} \sim 2.46 .
$$

The comparison with another special case shows that the above sequence of rectangles is far from being a minimizing sequence. Indeed, let $T$ denote the equilateral triangle with vertices $(0,0),(1,0)$, and $(1 / 2, \sqrt{3} / 2)$. Then (see for instance [20])

$$
u_{T}(x, y)=\sin \left(\frac{4 \pi y}{\sqrt{3}}\right)-\sin \left(2 \pi\left(x+\frac{y}{\sqrt{3}}\right)\right)+\sin \left(2 \pi\left(x-\frac{y}{\sqrt{3}}\right)\right) \quad \text { and } \quad \lambda_{1}(T)=\frac{16 \pi^{2}}{3} .
$$

By direct computation one gets

$$
\int_{\partial T}\left|\nabla u_{T}\right|^{2} \mathrm{~d} \mathcal{H}^{1}=\frac{9}{2} \frac{16 \pi^{2}}{3}
$$

so that

$$
\frac{\lambda_{1}(T)^{3 / 2}}{\int_{\partial T}\left|\nabla u_{T}\right|^{2} \mathrm{~d} \mathcal{H}^{1}}=\frac{4 \pi}{\sqrt{3}} \frac{2}{9} \sim 1.61
$$

\section{LOCAL ANALYSIS}

In this section, we develop local concavity arguments in order to obtain qualitative properties of the optimal sets minimizing (or maximizing) functionals over $\mathcal{K}_{0}^{n}$. Since the Minkowski addition is not useful for performing local perturbations of shapes, we use the general framework of deformations by vector fields associated to the classical shape derivative tools, see [26] and references therein. The idea was introduced in [32,33], where the 
authors study the convexity constraint for planar shapes: they point out a local concavity behavior of the shape functionals, expressed via the second order shape derivative, which implies that optimal convex shapes are polygons. Roughly speaking, this tool allows to deal with the indecomposability concept in a local sense, which naturally leads to identify polygons with extremal sets.

Below, we extend this strategy in any dimension of the space and prove that under a suitable local concavity assumption for the shape functional, optimal convex sets need to be extremal in the sense that their Gauss curvature cannot be positive. We finally show that this strategy can be applied to a large class of isoperimetric problems, including the conjecture of Pólya and Szëgo.

\subsection{Local concavity of the shape functional}

For the convenience of the reader, we briefly remind the notion of first and second order shape derivatives, focusing on local $\mathcal{C}^{2}$ deformations, since they are the ones we use for our purpose. Let $\mathcal{O}$ be a collection of sets in $\mathbb{R}^{n}$ and $J: \mathcal{O} \rightarrow \mathbb{R}$ be a shape functional. If $U$ is a compact set in $\mathbb{R}^{n}$ and $\varepsilon \in(0,1)$, we denote $\Theta_{U, \varepsilon}=\left\{\theta \in \mathcal{C}^{2}\left(\mathbb{R}^{n}, \mathbb{R}^{n}\right) / \operatorname{Supp}(\theta) \subset U\right.$ and $\left.\|\theta\|_{2, \infty}<\varepsilon\right\}$ endowed with the $W^{2, \infty}$-norm.

Definition 4.1. Let $K \in \mathcal{O}$, and $U$ a compact set in $\mathbb{R}^{n}$. It is said that $J$ is shape differentiable at $K$ (in $U$ ) if there exists $\varepsilon>0$ such that $K_{\theta}:=(I d+\theta)(K) \in \mathcal{O}$ for all $\theta \in \Theta_{U, \varepsilon}$ and $\mathcal{J}_{K}: \theta \in \Theta_{U, \varepsilon} \mapsto J\left(K_{\theta}\right) \in \mathbb{R}$ is differentiable at 0 .

Similarly, $J$ is twice differentiable at $K$ (in $U$ ) if there exists $\varepsilon>0$ such that $\mathcal{J}_{K}$ is differentiable in $\Theta_{U, \varepsilon}$, and if $\mathcal{J}_{K}^{\prime}: \Theta_{U, \varepsilon} \rightarrow \Theta_{U, \varepsilon}^{\prime}$ is differentiable at 0 .

The following result refers to the structure of second order shape derivatives (see [26]), and underlines the fact that the normal component of the deformation field plays a fundamental role in the computation of the shape derivatives.

Proposition 4.2. Let $K \in \mathcal{O}$ and $\omega$ a relatively open region of class $\mathcal{C}^{3}$ contained in $\partial K$. Let $U \subset \mathbb{R}^{n}$ be a compact set such that $U \cap \partial K \subset \omega$. If $J$ is twice differentiable at $K$ (in $U$ ), then there exists a continuous bilinear form $l_{2}^{J}(K): \mathcal{C}^{1}(\omega) \times \mathcal{C}^{1}(\omega) \rightarrow \mathbb{R}$ such that,

$$
\forall \theta \in \Theta_{U} \text { normal in } \omega, \quad \mathcal{J}_{K}^{\prime \prime}(0) \cdot(\theta, \theta)=l_{2}^{J}(K) \cdot(\varphi, \varphi),
$$

where $\varphi:=\theta \cdot \nu_{K}$ is the restriction to $\omega$ of the normal component of $\theta$ (and $\Theta_{U}=$ $\left.\left\{\theta \in \mathcal{C}^{2}\left(\mathbb{R}^{n}, \mathbb{R}^{n}\right) / \operatorname{Supp}(\theta) \subset U\right\}\right)$.

We are now able to state the main result of this section. Roughly speaking it states that, if the second order derivative of the shape functional satisfies a coercivity-like property, its minimizers among convex bodies cannot contain smooth regions with positive Gauss curvature in their boundary.

Below we denote by $|\cdot|_{H^{1}(\omega)}$ the classical semi-norm of $H^{1}(\omega)$, and by $\|\cdot\|_{H^{\frac{1}{2}}(\omega)}$ the classical norm of $H^{\frac{1}{2}}(\omega)$.

Theorem 4.3. Let $K^{*}$ be a minimizer for a functional $J: \mathcal{K}_{0}^{n} \rightarrow \mathbb{R}$. Assume that $\partial K^{*}$ contains a relatively open set $\omega$ of class $\mathcal{C}^{3}$ such that, for every compact set $U$ with $(U \cap \partial K) \subset \omega, J$ is twice differentiable at $K^{*}$ in $U$ and the bilinear form $l_{2}^{J}\left(K^{*}\right)$ satisfies

$$
\forall \varphi \in \mathcal{C}_{c}^{\infty}(\omega), \operatorname{Supp}(\varphi) \subset U, \quad l_{2}^{J}\left(K^{*}\right) \cdot(\varphi, \varphi) \leq-C_{1}|\varphi|_{H^{1}(\omega)}^{2}+C_{2}\|\varphi\|_{H^{\frac{1}{2}}(\omega)}^{2}
$$

for some constants $C_{1}>0, C_{2} \in \mathbb{R}$. Then $G_{K^{*}}=0$ on $\omega$ (where $G_{K^{*}}$ is the Gauss curvature).

Remark 4.4. The presence of regions with vanishing Gauss curvature on the boundary of an optimal convex set was already observed in [7] for the Newton problem of minimal resistance (see also [31]). A fundamental difference is that, in our framework, such a vanishing property of Gauss curvature is obtained by using the coercivity-like property (4.1) as a key argument, since the kind of shape functionals we deal with depend on PDE's ( $c f$. Thm. 4.5 below). 
Proof. Assume by contradiction that $G_{K^{*}}$ is positive at some point of $\omega$. By continuity, without loosing generality one may assume that $G_{K^{*}}>0$ on $\omega$.

Let $\varphi \in \mathcal{C}_{c}^{\infty}(\omega)$, and let $V \in \Theta_{U}$ be an extension of $\varphi \nu_{K} \in \mathcal{C}_{c}^{2}\left(\omega, \mathbb{R}^{n}\right)$ to $\mathbb{R}^{n}$, with compact support $U$ and such that $(U \cap \partial K) \subset \omega$. All the principal curvatures of $K^{*}$ being positive in $\omega$, by a continuity argument the principal curvatures of $K_{t}:=(I d+t V)\left(K^{*}\right)$ are still positive for $t$ small enough. Therefore $K_{t}$ is still a convex set, and one can write

$$
J\left(K^{*}\right) \leq J\left(K_{t}\right) \quad \text { for } t \text { small. }
$$

Therefore, the second optimality conditions gives

$$
0 \leq l_{2}^{J}\left(K^{*}\right) \cdot(\varphi, \varphi)
$$

and so, using the coercivity property of $l_{2}^{J}$, we get

$$
\forall \varphi \in \mathcal{C}_{c}^{\infty}(\omega), \quad|\varphi|_{H^{1}(\omega)}^{2} \leq \frac{C_{2}}{C_{1}}\|\varphi\|_{H^{\frac{1}{2}}(\omega)}^{2} .
$$

Relying on the density of $\mathcal{C}_{c}^{\infty}(\omega)$ in $H_{0}^{1}(\omega)$, we get the continuous imbedding of $H_{0}^{\frac{1}{2}}(\omega)$ in $H_{0}^{1}(\omega)$, which is a contradiction.

\subsection{Extremal problems under surface constraint}

Theorem 4.5. Let $K^{*}$ be a minimizer over $K_{0}^{n}$ for a shape functional of the kind

$$
\mathcal{E}(K):=\frac{F(K)}{S(K)}
$$

where $F(K)=f\left(\operatorname{Vol}(K), \lambda_{1}(K), \tau(K), \operatorname{Cap}(K)\right)$ for some $\mathcal{C}^{2}$ function $f: \mathbb{R}^{4} \rightarrow(0,+\infty)$.

If $\partial K^{*}$ contains a relatively open set $\omega$ of class $\mathcal{C}^{3}$, then $G_{K^{*}}=0$ on $\omega$.

Remark 4.6. When $n=3$ and $F(K)=\operatorname{Cap}(K)^{2}$, the Pólya-Szëgo conjecture claims that the minimizer for (4.2) is the planar disk. As a consequence of Theorem 4.5, we obtain that every set $K \in \mathcal{K}_{0}^{3}$ whose boundary contains a smooth region with positive Gauss curvature cannot be a minimizer.

Proof of Theorem 4.5. If $K^{*}$ minimizes $\mathcal{E}$ in $\mathcal{K}_{0}^{n}$, then it also minimizes the functional $K \mapsto J(K):=F(K)-$ $\beta S(K)$ for $\beta:=\frac{F\left(K^{*}\right)}{S\left(K^{*}\right)}>0$. The conclusion of Theorem 4.5 is then a direct consequence of Theorem 4.3 and of the following lemma: indeed, we get

$$
\begin{aligned}
\forall \varphi \in \mathcal{C}_{c}^{\infty}(\omega), \quad l_{2}^{J}\left(K^{*}\right) \cdot(\varphi, \varphi) & \leq-\beta c_{1}|\varphi|_{H^{1}(\omega)}^{2}+c_{2}\|\varphi\|_{H^{\frac{1}{2}}(\omega)}^{2}-\beta c_{2}^{\prime}\|\varphi\|_{L^{2}(\omega)}^{2} \\
& \leq-\beta c_{1}|\varphi|_{H^{1}(\omega)}^{2}+\left(c_{2}+\left|\beta c_{2}^{\prime}\right|\right)\|\varphi\|_{H^{\frac{1}{2}}(\omega)}^{2}
\end{aligned}
$$

with $\beta c_{1}>0$, and $c_{2}$ is obtained differentiating twice $f\left(\operatorname{Vol}(K), \lambda_{1}(K), \tau(K), \operatorname{Cap}(K)\right)$.

Lemma 4.7. Let $F(K)$ be any of the shape functionals $\operatorname{Vol}(K), \tau(K), \lambda_{1}(K), \operatorname{Cap}(K)$. Let $K$ be a bounded open set, and let $\omega \subset \partial K$ be a relatively open region of class $\mathcal{C}^{3}$. For any compact set $U$ such that $(U \cap \partial K) \subset \omega$, $F$ and $S$ are twice differentiable in $U$, and

$$
\forall \varphi \in \mathcal{C}_{c}^{2}(\omega), \operatorname{Supp}(\varphi) \subset U, \quad\left|l_{2}^{F}(K) \cdot(\varphi, \varphi)\right| \leq c_{2}\|\varphi\|_{H^{\frac{1}{2}(\omega)}}^{2} \text { and } l_{2}^{S}(K) \cdot(\varphi, \varphi) \geq c_{1}|\varphi|_{H^{1}(\omega)}^{2}+c_{2}^{\prime}\|\varphi\|_{L^{2}(\omega)}^{2}
$$

for some constants $c_{1}>0, c_{2}, c_{2}^{\prime} \in \mathbb{R}$. 
Proof. Except for the Newtonian capacity, the computations of the second order shape derivatives can be found in [26]. In our framework, the only difference is that we assume only the smoothness of $\omega$, where the local perturbations are performed. This does not affect the results of [26].

For the surface area, one has

$$
l_{2}^{S}(K) \cdot(\varphi, \varphi)=\int_{\partial K}\left|\nabla_{\tau} \varphi\right|^{2}+\left[H^{2}-\operatorname{Tr}\left({ }^{t} D_{\tau} \nu \cdot D_{\tau} \nu\right)\right] \varphi^{2},
$$

where $H$ is the mean curvature, well defined on the support of $\varphi \subset \omega$. This easily gives:

$$
l_{2}^{S}(K) \cdot(\varphi, \varphi) \geq|\varphi|_{H^{1}(\omega)}^{2}+c_{2}^{\prime}\|\varphi\|_{L^{2}(\omega)}^{2}
$$

where $c_{2}^{\prime}=\min _{\omega \cap U}\left[H^{2}-\operatorname{Tr}\left({ }^{t} D_{\tau} \nu \cdot D_{\tau} \nu\right)\right]$.

For Vol, $\lambda_{1}$, and $\tau$, formulas given in [26] easily give the $H^{\frac{1}{2}}$-continuity property.

Let us provide a complete proof of this result for the Newtonian capacity, by following the same lines as in [19].

For $\theta \in \Theta_{U, \varepsilon}$ with $\varepsilon$ small enough, we denote $K_{\theta}=(I d+\theta)(K), u_{\theta}=u_{K_{\theta}}$, and $v_{\theta}=u_{\theta} \circ(I d+\theta)$. We also denote by $\mathcal{D}$ (respectively $\mathcal{D}_{0}$ ) the closure of the space of smooth functions compactly supported in $\mathbb{R}^{3} \backslash K$ (respectively in $\mathbb{R}^{3} \backslash \bar{K}$ ) with respect to the Dirichlet norm $u \mapsto \int_{\mathbb{R}^{3} \backslash K}|\nabla u|^{2}$.

If we introduce $\psi \in \mathcal{C}_{c}^{\infty}\left(\mathbb{R}^{3}\right)$ with

$$
0 \leq \psi \leq 1, \quad \psi \equiv 1 \text { on an open neighborhood of } \bar{K},
$$

since $\Delta\left(\psi-u_{\theta}\right)=\Delta \psi$ on $K_{\theta}, w_{\theta}:=\psi \circ(I d+\theta)-v_{\theta}$ is a variational solution of

$$
w_{\theta} \in \mathcal{D}_{0}, \quad-\nabla \cdot\left(A(\theta) \nabla w_{\theta}\right)=[f \circ(I d+\theta)] J_{\theta},
$$

where

$$
f=\Delta \psi, \quad A(\theta)=J_{\theta}(I d+D \theta)^{-1}\left(I d+{ }^{t} D \theta\right)^{-1}, \quad J_{\theta}=\operatorname{det}(I d+D \theta) .
$$

Now, we consider the mapping

$$
F:(\theta, w) \in \Theta_{U} \times\left(\mathcal{D}_{0} \cap H^{2}\left(\left(\mathbb{R}^{3} \backslash K\right) \cap U\right)\right) \mapsto-\nabla \cdot(A(\theta) \nabla w)-[f \circ(I d+\theta)] J_{\theta} \in \mathcal{D}_{0}^{\prime} \cap L^{2}\left(\left(\mathbb{R}^{3} \backslash K\right) \cap U\right) .
$$

We check as in $[26]$ that $F$ is $\mathcal{C}^{\infty}$ and that

$$
D_{w} F\left(0, w_{0}\right)=-\Delta: \mathcal{D}_{0} \cap H^{2}\left(\left(\mathbb{R}^{3} \backslash K\right) \cap U\right) \rightarrow \mathcal{D}_{0}^{\prime} \cap L^{2}\left(\left(\mathbb{R}^{3} \backslash K\right) \cap U\right)
$$

is an isomorphism (by Lax-Milgram's Theorem and classical regularity results). By the implicit function Theorem, and unicity in (4.3), it follows that $\theta \in \Theta_{U} \mapsto w_{\theta} \in \mathcal{D}_{0} \cap H^{2}\left(\left(\mathbb{R}^{3} \backslash K\right) \cap U\right)$ is $\mathcal{C}^{\infty}$ around $\theta=0$, and studying the differentiability of $\theta \mapsto(I d+\theta)^{-1}$ and the composition operator (see [26], Prop. 5.3.10, Exercise 5.2, and [34]), one deduce that $\theta \in \Theta_{U} \mapsto u_{\theta}=\psi-w_{\theta} \circ(I d+\theta)^{-1} \in L_{l o c}^{2}\left(\mathbb{R}^{3}\right)$ is also twice differentiable around 0 .

Let us now compute the second order derivative of the Newtonian capacity: let $\varphi \in \mathcal{C}^{\infty}(\omega), \operatorname{Supp}(\varphi) \subset U$, and let $V \in \Theta_{U}$ be an extension to $\mathbb{R}^{n}$ of $\varphi \nu_{K}$, with compact support $U$ such that $U \cap \partial K \subset \omega$. For $t$ small, we denote $K_{t}=(I d+t V)(K), u_{t}=u_{K_{t}}$, and $v_{t}=u_{t} \circ(I d+t V)$; we get that for small enough $t$ such that $\partial K_{t} \subset \operatorname{Supp}(\psi)$,

$$
\operatorname{Cap}\left(K_{t}\right)=\int_{\mathbb{R}^{3} \backslash K_{t}}\left|\nabla u_{t}\right|^{2}=-\int_{\partial K_{t}} \frac{\partial u_{t}}{\partial \nu_{t}}=-\int_{\partial K_{t}} \psi \frac{\partial u_{t}}{\partial \nu_{t}}=-\int_{\mathbb{R}^{3} \backslash K_{t}} u_{t} \Delta \psi=-\int_{\mathbb{R}^{3}} u_{t} \Delta \psi
$$


and so $\frac{\mathrm{d}^{2}}{\mathrm{~d} t^{2}} \operatorname{Cap}\left(K_{t}\right)_{\mid t=0}=-\int_{\mathbb{R}^{3}} u^{\prime \prime} \Delta \psi=\int_{\mathbb{R}^{3} \backslash K} u^{\prime \prime} \Delta(u-\psi)=\int_{\partial K} u^{\prime \prime} \frac{\partial u}{\partial \nu}$ with

$$
\begin{aligned}
& \Delta u^{\prime}=0 \text { in } \mathbb{R}^{3} \backslash \bar{K}, \quad u^{\prime}+\nabla u \cdot V=0 \text { on } \partial K, \\
& \Delta u^{\prime \prime}=0 \text { in } \mathbb{R}^{3} \backslash \bar{K}, \quad u^{\prime \prime}+2 \nabla u^{\prime} \cdot V+\left(D^{2} u \cdot V\right) \cdot V \text { on } \partial K,
\end{aligned}
$$

where the boundary conditions are obtained when we differentiate $u_{t}(x+t V(x))=0, \forall x \in \partial K$ (since $V_{\mid \partial K}$ is supported in $\omega$, these expressions are well defined, since $u$ is regular enough up to $\omega$ ).

Let us denote by $R: f \in H^{\frac{1}{2}}(\partial K) \longmapsto z=R(f) \in \mathcal{D}$ such that $\left\{\begin{aligned} \Delta z=0 & \text { in } \mathbb{R}^{3} \backslash \bar{K} \\ z=f & \text { on } \partial K\end{aligned}\right.$ which is a continuous operator. Then

$$
\begin{aligned}
\left|\frac{\mathrm{d}^{2}}{\mathrm{~d} t^{2}} \operatorname{Cap}\left(K_{t}\right)_{\mid t=0}\right| & =\left|-\int_{\partial K}\left[2 \varphi \frac{\partial u}{\partial \nu} \frac{\partial R\left(-\varphi \frac{\partial u}{\partial \nu}\right)}{d \nu}+\varphi^{2} \frac{\partial u}{\partial \nu}\left(D^{2} u \cdot \nu\right) \cdot \nu\right]\right| \\
& =\left.\left|\int_{K} 2\right| \nabla R\left(-\varphi \frac{\partial u}{\partial \nu}\right)\right|^{2}+\int_{\partial K} \varphi^{2} \frac{\partial u}{\partial \nu}\left(D^{2} u \cdot \nu\right) \cdot \nu \mid \\
& \leq C\left(\left|R\left(\varphi \frac{\partial u}{d \nu}\right)\right|_{H^{1}(K)}^{2}+\|\varphi\|_{L^{2}(\omega)}^{2}\right) \\
& \leq c_{2}\|\varphi\|_{H^{\frac{1}{2}}(\omega)}^{2} .
\end{aligned}
$$

Acknowledgements. Part of the work was supported by the project ANR-09-BLAN-0037 Geometric analysis of optimal shapes (GAOS) financed by the French Agence Nationale de la Recherche (ANR).

\section{REFERENCES}

[1] A.D. Alexandrov, Zur theorie der gemischten volumina von konvexen korpern III, Mat. Sb. 3 (1938) $27-46$.

[2] V. Alexandrov, N. Kopteva and S.S. Kutateladze, Blaschke addition and convex polyedra. preprint, arXiv:math/0502345 (2005).

[3] C. Bianchini and P. Salani, Concavity properties for elliptic free boundary problems. Nonlinear Anal. 71 (2009) $4461-4470$.

[4] C. Borell, Capacitary inequalities of the Brunn-Minkowki type. Math. Ann. 263 (1983) 179-184.

[5] C. Borell, Greenian potentials and concavity. Math. Ann. 272 (1985) 155-160.

[6] H. Brascamp and E. Lieb, On extension of the Brunn-Minkowski and Prékopa-Leindler inequality, including inequalities for $\log$ concave functions, and with an application to diffision equation. J. Funct. Anal. 22 (1976) 366-389.

[7] F. Brock, V. Ferone and B. Kawohl, A Symmetry Problem in the Calculus of Variations, Calc. Var. Partial Differential Equations 4 (1996) 593-599.

[8] E.M. Bronshtein, Extremal H-convex bodies. Sibirsk Mat. Zh. 20 (1979) 412-415.

[9] D. Bucur, G. Buttazzo and A. Henrot, Minimization of $\lambda_{2}(\Omega)$ with a perimeter constraint. Indiana Univ. Math. J. 58 (2009) 2709-2728.

[10] L. Caffarelli, D. Jerison and E. Lieb, On the case of equality in the Brunn-Minkowski inequality for capacity. Adv. Math. 117 (1996) 193-207.

[11] S. Campi and P. Gronchi, On volume product inequalities for convex sets. Proc. Amer. Math. Soc. 134 (2006) $2393-2402$.

[12] A. Colesanti, Brunn-Minkowski inequalities for variational functionals and related problems. Adv. Math. 194 (2005) 105-140.

[13] A. Colesanti and P. Cuoghi, The Brunn-Minkowski inequality for the $n$-dimensional logarithmic capacity. Potential Anal. 22 (2005) 289-304

[14] A. Colesanti and M. Fimiani, The Minkowski problem for the torsional rigidity. Indiana Univ. Math. J. 59 (2010) $1013-1040$.

[15] A. Colesanti and P. Salani, The Brunn-Minkowski inequality for p-capacity of convex bodies. Math. Ann. 327 (2003) 459-479.

[16] G. Crasta and F. Gazzola, Some estimates of the minimizing properties of web functions, Calc. Var. Partial Differential Equations 15 (2002) 45-66. 
[17] G. Crasta, I. Fragalà and F. Gazzola, On a long-standing conjecture by Pólya-Szegö and related topics. Z. Angew. Math. Phys. 56 (2005) 763-782.

[18] A. Figalli, F. Maggi and A. Pratelli, A refined Brunn-Minkowski inequality for convex sets. Ann. Inst. Henri Poincaré Anal. Non Linéaire 26 (2009) 2511-2519.

[19] I. Fragalà, F. Gazzola and M. Pierre, On an isoperimetric inequality for capacity conjectured by Pólya and Szegö. J. Differ. Equ. 250 (2011) 1500-1520.

[20] P. Freitas, Upper and lower bounds for the first Dirichlet eigenvalue of a triangle, Proc. Am. Math. Soc. 134 (2006) $2083-2089$.

[21] R. Gardner, The Brunn-Minkowski inequality. Bull. Am. Math. Soc. (N.S.) 39 (2002) 355-405.

[22] R.J. Gardner and D. Hartenstine, Capacities, surface area, and radial sums, Adv. Math. 221 (2009) 601-626.

[23] P.R. Goodey and R. Schneider, On the intermediate area functions of convex bodies. Math. Z. 173 (1980) $185-194$.

[24] E. Grinberg and G. Zhang, Convolutions, transforms, and convex bodies. Proc. London Math. Soc. 78 (1999) 77-115.

[25] H. Hadwiger, Konkave Eikörperfunktionale. Monatsh. Math. 59 (1955) 230-237.

[26] A. Henrot and M. Pierre, Variation et Optimisation de Formes: une analyse géométrique, Mathématiques et Applications 48. Springer (2005).

[27] D. Jerison, A Minkowski problem for electrostatic capacity. Acta Math. 176 (1996) 1-47.

[28] D. Jerison, The direct method in the calculus of variations for convex bodies. Adv. Math. 122 (1996) $262-279$.

[29] S.S. Kutateladze, One functional-analytical idea by Alexandrov in convex geometry. Vladikavkaz. Mat. Zh. 4 (2002) 50-55.

[30] S.S. Kutateladze, Pareto optimality and isoperimetry. preprint, arXiv:0902.1157v1 (2009).

[31] T. Lachand-Robert and M.A. Peletier, An example of non-convex minimization and an application to Newton's problem of the body of least resistance. Ann. Inst. Henri Poincaré 18 (2001) 179-198.

[32] J. Lamboley and A. Novruzi, Polygons as optimal shapes with convexity constraint. SIAM J. Control Optim. 48 (2009/10) $3003-3025$

[33] J. Lamboley, A. Novruzi and M. Pierre, Regularity and singularities of optimal convex shapes in the plane, preprint (2011).

[34] M. Lanza de Cristoforis, Higher order differentiability properties of the composition and of the inversion operator. Indag. Math. $N S \mathbf{5}$ (1994) 457-482.

[35] G. Pólya and G. Szegö, Isoperimetric Inequalities in Mathematical Physics, Annals of Mathematics Studies 27. Princeton University Press, Princeton, N.J. (1951).

[36] Ch. Pommerenke, Univalent functions. Vandenhoeck and Ruprecht, Göttingen (1975).

[37] P. Salani, A Brunn-Minkowski inequality for the Monge-Ampère eigenvalue. Adv. Math. 194 (2005) 67-86.

[38] R. Schneider, Eine allgemeine Extremaleigenschaft der Kugel. Monatsh. Math. 71 (1967) 231-237.

[39] R. Schneider, Convex bodies: the Brunn-Minkowski theory. Cambridge Univ. Press (1993). 